\title{
Automatically Generated Landmark-enhanced Navigation Instructions for Blind Pedestrians
}

\author{
Jan Balata*, Zdenek Mikovec $^{*}$, Petr Bures ${ }^{\dagger}$, Eva Mulickova ${ }^{\ddagger}$ \\ ${ }^{*}$ Faculty of Electrical Engineering, Czech Technical University in Prague, Czech Republic \\ ${ }^{\dagger}$ Faculty of Transportation Sciences, Czech Technical University in Prague, Czech Republic \\ $\ddagger$ Central European Data Agency, a. s. (CEDA), Czech Republic \\ balatjan@fel.cvut.cz,xmikovec@fel.cvut.cz
}

\begin{abstract}
Visual impairment limits a person mainly in ability to move freely and independently. Even with many navigation aids and tools currently on the market, almost one third of the visually impaired do not travel independently without a guide, and human-prepared landmark-enhanced itineraries of the route are the most useful. We designed a system which based on a specific efficiently collected geographical data generates human-like landmark-enhanced navigation instructions. The studies we conducted (quantitative $n=16$, qualitative $n=6$ ) proved usability and efficiency of the system. Further we provide set of design recommendations to increase the usability of the system along with specific examples of usage with particular landmarks.
\end{abstract}

\section{INTRODUCTION}

T HE ability to travel independently is required for satisfactory level of quality of life and self confidence. Visual impairment limits mainly person's mobility and reduces travel-related activities [1]. This can lead to loss of work, friends, and hobbies and eventually to worsening psychical condition of a person. Even though visually impaired people undergo special training of navigation and orientation skills, $30 \%$ of them never leave home alone without sighted guide [2], [3] and this number remains stable for last twenty five years.

The mobility of a person is mainly influenced by the efficiency of the wayfinding process [4]. This process consists of two parts. First, environment sensing such as avoiding obstacles and hazards. Second, the navigation to remote destination. Both parts of the wayfinding process can be supported by navigation aids, which assist the visually impaired pedestrian. The basic criteria for evaluation of navigation aids are safety, efficiency, and stress level, as defined by Armstrong [5].

Currently, this problem is solved by means of car navigation systems, which are not suitable for visually impaired, or better by assistance of orientation and mobility specialists. These specialists can prepare route itinerary to remote destination for visually impaired person for a particular route in advance and provide it in a form of a itinerary. This solution suffers from time requirements (it has to be prepared in advance) and rigidity (there is no option to change the route at user's will).

In our work we focused on developing efficient navigation aid which supports navigation to remote destination. We aimed to generate route itineraries similar to those prepared by orientation and mobility specialist. By using sophisticated data structures and algorithms we addressed the issue of time requirements (the itinerary is created immediately) and rigidity (user can select whichever origin and destination s/he want).

To compare our solution to state-of-art electronic navigation systems we developed two versions of the navigation system. The first version (Landmark), with itineraries enhanced by landmarks. The second version (Metric), simulating current metric-based navigation systems. For fair comparison of both conditions, Metric version also used pedestrian network for routing (sidewalks, crossings). Further, we provide the insights from qualitative study conducted on Landmark version.

\section{RELATED WORK}

\section{A. Pedestrian Navigation}

Successful navigation and orientation in a space depends on building of spatial knowledge about the given environment. Siegel and White [6] define three levels of spatial knowledge. These levels are: landmark knowledge, route knowledge, and overview knowledge.

Relations between objects represent an overview knowledge. These relations may be represented by angles or distances between objects not necessarily located or related to the route itself.

The landmarks (representing landmark knowledge) represent the most frequently-used category of navigation cues used by pedestrians [7] (unlike distance, junctions or road type). Ross et al. [8] have shown that inclusion of landmarks within pedestrian route itinerary increased user confidence and reduced navigation errors. Findings of Ross et al. [8] extend also to voice-only navigation [9], where inclusion of landmarks was clearly more prefered by participants

There are also some experimental designs of navigation systems, which rely primarily on landmarks to navigate users from origin A to destination B, e.g. Millonig and Schechtner [10].

In our system we aim to enhance metric-based navigation instructions with carefully selected landmarks suitable for navigation of visually impaired people. 


\section{B. Orientation and Navigation of Blind}

In large spaces visually impaired pedestrians use different cognitive strategies from sighted ones, based on egocentric frames [11], [12]. Visually impaired people have to memorize large amount of information [13] in sequential order [11] while traveling. Fortunately a study by Raz et al. [14] has shown that congenitally blind people are better in both item memory and serial memory than sighted people. Their memory skills are outstanding namely for long sequences of information. Bradley and Dunlop [15] also discovered that blind people were significantly faster with verbal guidance from blind navigator than from sighted one.

Many navigation aids for visually impaired pedestrians have been developed. Some of the aids use special sensors to identify object along the route like cameras [16], or RFID based white canes [17]. Other navigation aids are based on concepts described in [18], and use some kind of position system (e.g. GPS) in combination with geographical information system (GIS) to navigate the pedestrian, e.g. Ariadne GPS, BlindSquare. Navigation aids based on special interaction techniques, e.g. an auditory display [4] or a tactile compass [19], has also been developed.

The navigation systems based on major GIS (e.g. OpenStreetMap) typically suffer from inappropriate level of detail (missing sidewalks, leading lines, slopes of sidewalk), ambiguity (inadequate description of pedestrian crossing so it cannot be located without sight), or they do not optimize routing algorithm to meet specific abilities of visually impaired people (e.g. inability to cross large open spaces).

In cooperation with major national data provider we focus on development of sustainable and scalable GIS with deeply modified data structure (see subsection Geographical Information Database), which allows us to generate specific landmarks for navigation of visually impaired people.

\section{LANDMARK-ENHANCED INSTRUCTIONS GENERATION}

To implement feasible solution to generate a landmark-enhanced navigation instructions, we need specially modified GIS which is capable of representing special features of the urban environment. Further we need algorithms, which use the landmarks and their parameters to generate route itinerary in a natural language. In our case each route itinerary is composed of navigation instructions for each segment of a route (typically part of a route from corner to corner, corner to crossing, etc.). For each navigation instruction we have chosen the following structure: environment description and action that should be performed by the blind pedestrian.

\section{A. Geographical Information Database}

Generally, we distinguish line, point and area features. Line features are tied to large part of pedestrian segment (e.g. geometry representation of a sidewalk in GIS) and represent their properties (like slope, surface quality) or phenomena along the segment (e.g. parking cars, railings). Point features describe phenomena that covers very small part of pedestrian

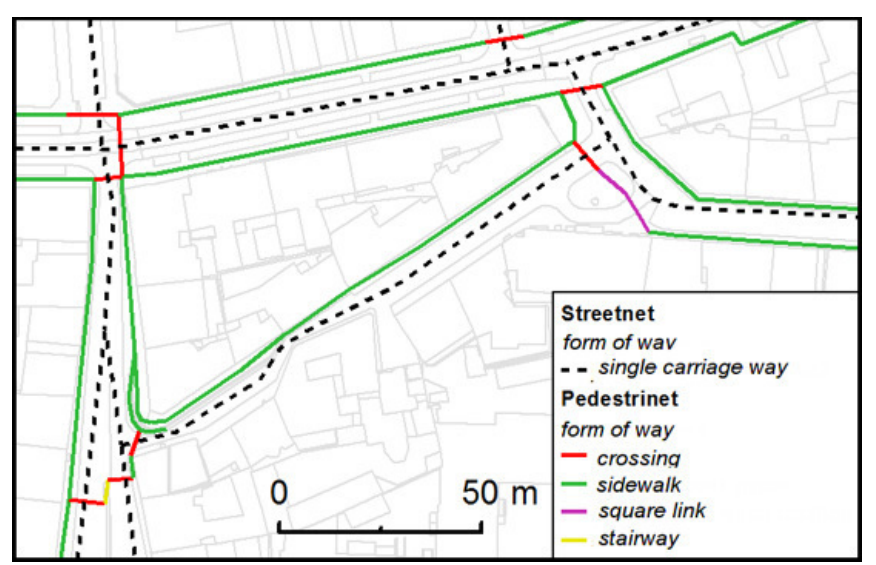

Fig. 1: Comparison of StreetNet and PedestriNet - background data: Digital city map - Map of town utilities (source: Open data).

segment (typically less than $3 \mathrm{~m}$ ), they act as barriers or landmarks (e.g. crossing entry points, narrowings, steps, corners). Area features are landmarks extending over a certain area like traffic noise (busy streets, certain types of public transport, etc.).

The nature of those new features and the range of descriptive information reflect the needs of both visually impaired and wheelchair users. The complete data model was proposed in cooperation with Central European Data Agency, a.s. (CEDA), Faculty of Transportation Science and Faculty of Electrical Engineering (Czech Technical University in Prague) within project ROUTE4ALL. Data model was implemented as ArcGIS Geodatabase ${ }^{1}$.

Pedestrian segment network. To be able to collect desired landmarks and their properties, the existence of the pedestrian segment network is essential. The national provider of digital vector road geodatabase StreetNet, thus proposed a new product PedestriNet that represents all paths designated for pedestrians. StreetNet is currently used for pedestrian navigation for users without disabilities. For disabled users it is not suitable due to simplified representation of crossings and sidewalks that does not reflect real topology of pedestrian network.

PedestriNet is characterized by very high positional accuracy - it represents footpaths in their real position in the level of Map of town utilities (see Fig 1) and maintain the topology of the pedestrian network. It covers special pedestrian segments like sidewalk, crossing, square link (a sidewalk crossing large open area of a quare) as well as pedestrian segments already represented in StreetNet database (e.g. pedestrian zone, walkway, gallery).

Geodatabase. All features of geodatabase include reference information to PedestriNet - ID of segment, orientation towards it (left/right/on). Thus they can be used for routing along the pedestrian network and generation of route itinerary.

\footnotetext{
${ }^{1}$ ArcGIS - http://www.arcgis.com/
} 
To minimise data collection costs it is necessary to use existing data as much as possible. City municipalities administer Map of town utilities in high accuracy level that may be used to locate some features (e.g. stairways, corners) or to derive some properties (e.g. width of sidewalk). Unfortunately, these maps are mostly CAD drawings and therefore full automation of data processing is impossible. Further, Land-use map can be used to derive land-use of adjacent footpath area, digital terrain model can be used to calculate slope of footpath, etc. Another source of information may be actual video recordings. Field survey remains necessary to derive quantitative data for wheelchair users (e.g. height of curbs) and to verify collected data. Here, community reports may be of high importance.

Prototype of the geodatabase that is used for validation and testing covers area of about $1 \mathrm{~km}^{2} / 52 \mathrm{~km}$ of pedestrian segments. This area took around 5 man/days to collect data and fill the geodatabase.

\section{B. Navigation Instruction Structure}

As described above, each navigation instruction is composed of environment description and action that should be performed by the blind pedestrian (similarly to [20]).

The environment description is generated from street names, addresses, corners, and crossings. The action is generated from geometry, street names, corners, slopes, land-use, and point features.

Table I shows how each navigation instruction is composed on a short 5 segment route itinerary with one crossing.

\section{Algorithm}

First a route is found by Dijkstra algorithm on PedestriNet graph. Then, for each segment, which is represented by vector data, we create a navigation instruction.

For generation of navigation instruction we find best matching sentence templates, which are selected based on a type of a segment, context (available metadata, adjacent segments, direction of the user, etc.), and priority (e.g. the template for corner is more prefered than the template for crossing) (see Listing 1).

An example for sentence template for environment description of a place "You are at a beveled corner of streets Odboru and Karlovo namesti." (see Listing 2).

In this way we created landmark-enhanced navigation instruction for blind pedestrians (Landmark), which were later compared with metric-based navigation instructions (Metric).

\section{Comparative Study}

In our experiment we raised the question whether the error rate is lower for Landmark condition than for Metric condition, and whether measured completion time is lower for Landmark condition than for Metric condition.

Further we investigated subjective judgement of the participants about the level of safety, comprehension, and ambiguity of the generated itineraries, along with qualitative observations.
Listing 1: Selecting best sentence template.

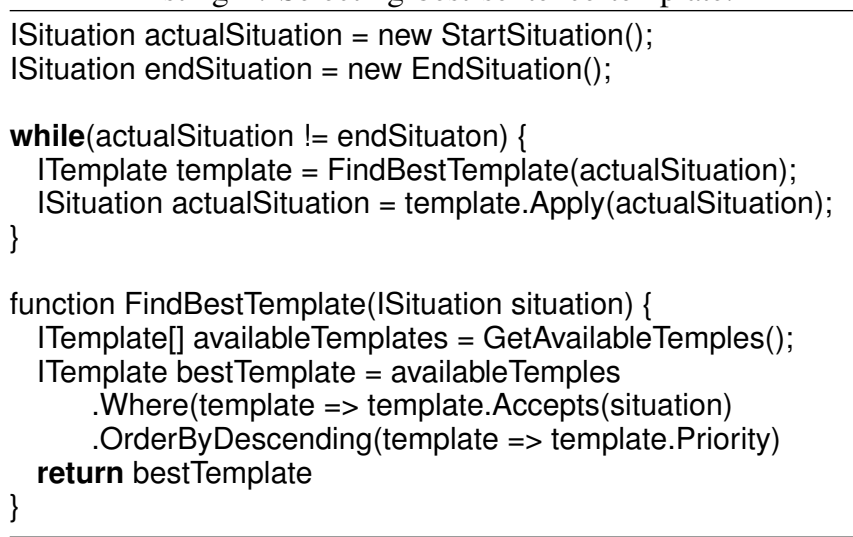

Listing 2: Application of a sentence template. class CornerDescriptionTemplate : ITemplate \{

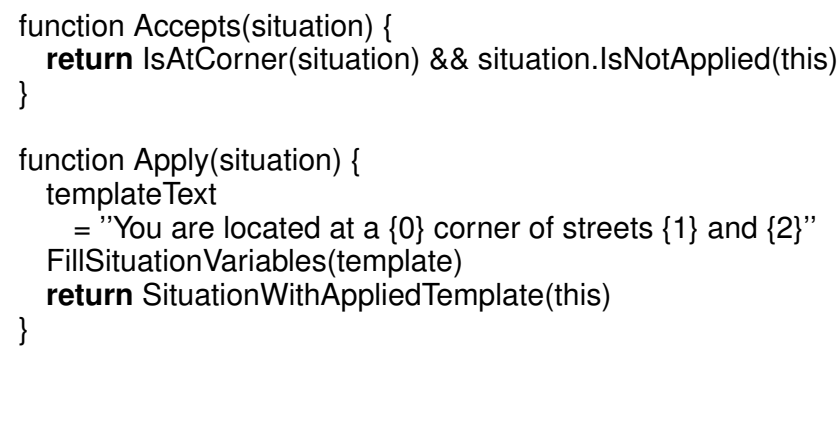

\section{A. Participants}

Sixteen visually impaired participants (10 female, 6 male) were recruited via e-mail leaflet sent to a group of our long term collaborators of our University. The participants in the experiment were aged from 23 to 66 years (mean $=35.75$, $S D=11.23)$. Eleven participants had Category 5 visual impairment (no light perception); 5 participants had Category 4 visual impairment (light perception) [21]; 4 participants were late blind, 12 participants were congenitally blind. All of the participants were native Czech speakers.

\section{B. Apparatus}

Routes. For our experiment, we selected two routes in city center outdoor environment. Environments for this type of experiment are usually real environments [9], [15] rather than artificial (lab) environments, though exceptions are possible [22]. The location of the routes was in quiet area in the city center of Prague, Czech republic (see Fig. 2). Both of the routes were approximately 350 meters long. Both of the routes consisted of 7 segments and 8 decision points (points where the participant changes his/her direction). They had the same number of turns and pedestrian crossings. Thus we consider the routes to be the same similar to [23]. 
TABLE I: Main building blocks used for automated generation of route itineraries.

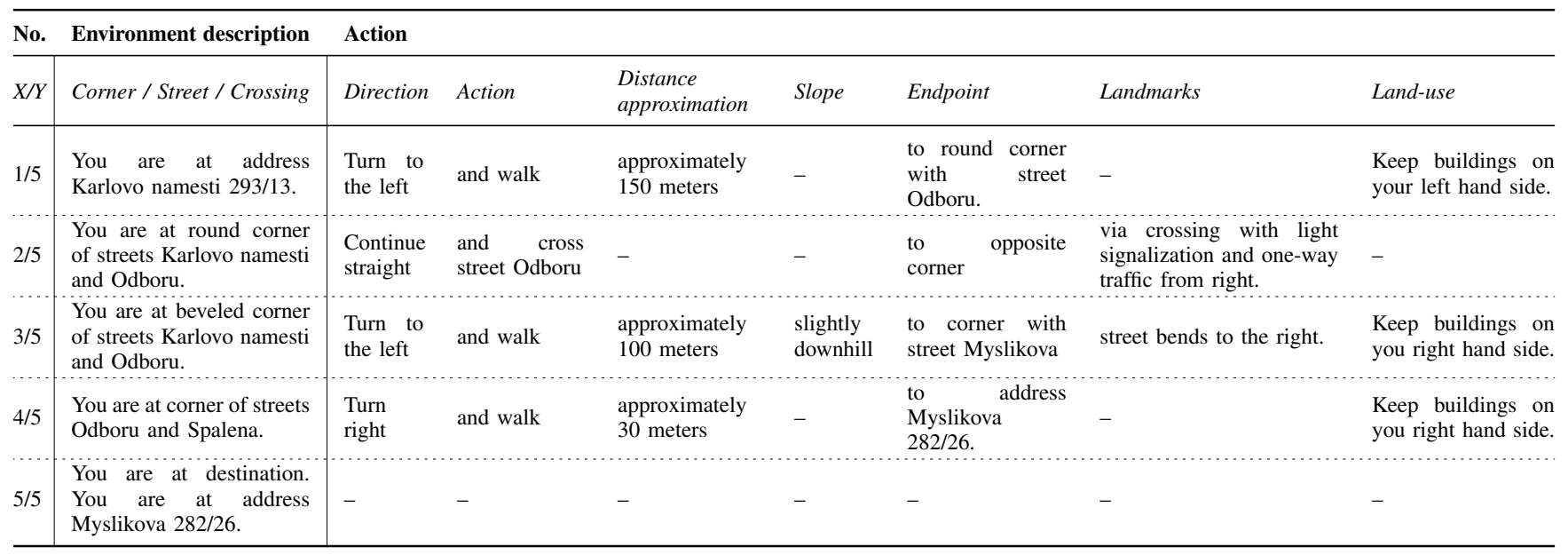

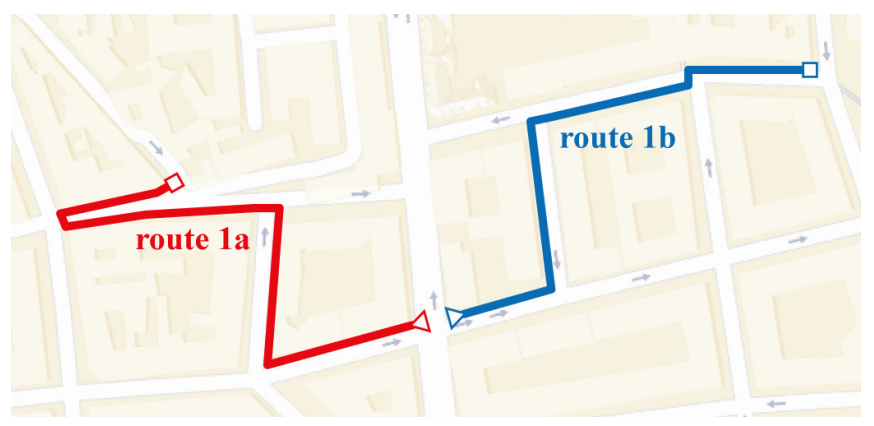

Fig. 2: Routes used in comparative study, the triangle depicts the beginning of a route, the square depicts destination of a route.

Equipment. The participant was equipped with a Nokia 6120 mobile phone with a lanyard which hung from his/her neck. In this way, the phone was protected from being dropped unintentionally, and the participant was able to release it and have an empty hand when needed, and s/he could also find it again quickly. The mobile phone was set to Czech language, and it was equipped with the MobileSpeak text-to-speech (TTS) screen reader application by CodeFactory.

Data collection. In each session, we recorded two video streams of the participant's activities. The first camera (GoPro Hero 3) recorded $1^{\text {st }}$ person view and was installed on a shoulder strap of the backpack that was carried by the participants during the session, while the second camera (Sony DLSR) recorded a $3^{\text {rd }}$ person view by the experimenter shadowing the participant.

\section{Procedure}

The experiment consisted of two walkthroughs of each route and it lasted around 1.5 hour. In the first walkthrough the experimenter guided the participants to the beginning of the first route, explained the purpose of the experiment to the participants, explained operation of the navigation application, and asked the participant to adjust the phone on a lanyard or to hold it in a hand, according to his/her own preference. The participant was asked to proceed as quickly and accurately as possible. The task was given as follows: "You have a meeting in Hostel Emma (for route A; Cafe Amandine for route B). To reach the destination use the navigation application. Proceed as if you were alone, but we will be watching for your safety from a distance." Than the participant started out.

After the first walkthrough the participant was returned to the start of the route and walked the route with the experimenter. The participants were retrospectively asked their about subjective judgement about level of safety ("Did the participant feel safe?"), comprehension ("Did the participant understand what to do?") and ambiguity ("Was the description of the environment ambiguous?") for each segment of a route (Likert scale 1-5 was used).

Then the experimenter took the participant to the start of the second route and proceeded same as on the first route. After the experiment the participant was debriefed and received their payments.

\section{Design}

The experiment was one factor (two levels) within subject design. The independent variable was itinerary quality (Landmark, Metric). The itinerary quality and route were balanced using a Latin square. The main measures were an error rate, defined as 0,1 if there was a navigation error (e.g. participant missed the turn, crossed the road on different place, etc.) on a particular segment of a route, and a completion time, calculated as time taken for traveling from start to destination of a route. For analysis of error rate and completion time we use confidence intervals (according to [24]).

\section{E. Results and Discussion}

The following subsections describe findings observed during the experiment. We collected the data from 16 sessions and based on the results we propose general design recommendations for creation of navigation instructions. 


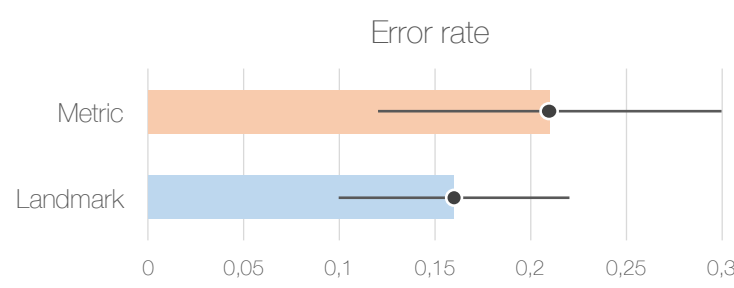

Fig. 3: Mean error rates with $95 \%$ confidence intervals for Landmark and Metric condition $(\mathrm{n}=16$, lower is better).

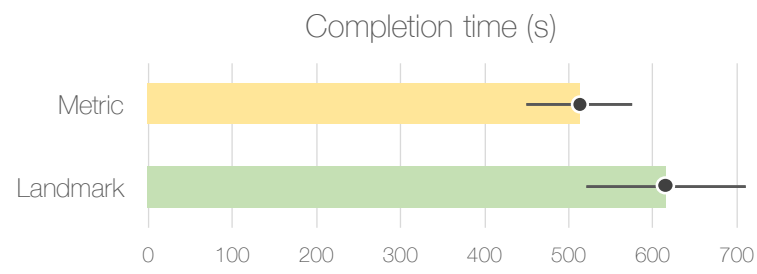

Fig. 4: Mean completion times with $95 \%$ confidence intervals for Landmark and Metric condition ( $\mathrm{n}=16$, lower is better).

Error rate. Fig. 3 provides evidence on error rates and $95 \%$ confidence intervals. It seems that error rate for Landmark (mean $=0.16,95 \% C I[0.10,0.22]$ ) was very similar as for Metric $($ mean $=0.21,95 \% C I[0.12,0.30]$ ) and the results are largely inconclusive concerning the difference between the test conditions, although with small favor for Landmark.

We could not decide whether the lack of difference in error rate was caused by random variables occuring during the experiment (see paragraph Random variables) or by selection of rather easy routes as described by participants.

Completion time. Fig. 4 provides evidence on completion times in seconds and $95 \%$ confidence intervals. It seem that completion time for Landmark (mean $=615.4$ seconds, $95 \% C I[520.89,709.99])$ is $1.2 \times$ higher on average than for Metric $($ mean $=513.00$ seconds, $95 \%$ CI [450.33, 575.67]) and the results show that there is an effect of the test conditions on completion time. The completion times ranged from 267 to 917 seconds for Landmark test condition and it ranged from 292 to 757 seconds for Metric test condition.

It cannot be decided whether the difference in completion times for Landmark and for Metric was caused by longer text in the navigation instructions (participant waited longer time to listen it whole) or by occurrence of random variables (sometimes participants stopped because they didn't feel secure, see paragraph Random variables). It would be necessary to repeat the experiment in more controlled conditions, however it would affect external validity of the experiment.

Subjective judgment. During the second walkthrough we asked the participant about their subjective judgment of each segment of a route. The results suggest that comprehension is higher for Landmark ( $85 \%$ of the participants strongly agree) than for Metric (65\% of the participants strongly agree).
Ambiguity and safety were evaluated similarly for both test conditions (see Fig. 5).

Further we asked the participant for the comments on navigation instructions for both conditions. In Landmark condition, the participants highlighted information about traffic direction or information about land-use. However, in both test conditions they had problems with lack of the corner descriptions and lack of endpoints at crossings. In both test conditions, the participants often confused individual navigation instructions with each other as they started similarly. In Metric condition, the participant lacked information about endpoints and they were surprised by very precise distances (precision up to meters; in Landmark distances are rounded to tens of meters) reported by the system ("I cannot tell how far did I went precisely", P07, participant P01 laughed about it).

Random variables. Similarly to Rehrl et al. [9] our measurement were influenced by random variables (unpredictable urban environment). Further we classify the problems we observed into 4 categories.

Collision with objects $-28 \times$. We observed that participants frequently collide with traffic signs, poles, beer gardens or parking-ticket machines. This seems as a common problems for the visually impaired pedestrians.

Interference of passerby people $-10 \times$. During the experiment we observed the participant from a distance (shadowing method). Sometimes passerby people stopped the participants and offered them help $(4 \times)$, however $6 \times$ they grabbed, dragged or guided the participant to some arbitrary chosen spot, which resulted in loss of orientation of the participant.

Disruption of the senses $-16 \times$. As a hearing is one of primary orientation and navigation senses for visually impaired pedestrians, its disruption strongly affects the wayfinding process. We observed following sources of hearing disruption: garbage disposal trucks, road cleaning trucks, and rain. Another case was problem with finger sensitivity and operation of our Nokia smartphone.

Stress $-2 \times$. One participant was anxious about the experiment even though we tried to calm him/her during briefing, it affected his/her performance ( $\mathrm{s} / \mathrm{he}$ proceeded much better towards the end of the experiment than in the beginning). Another case was when participant dropped his/her white cane ( $\mathrm{s} /$ he was immediately assisted and handed the cane).

Allover we counted 32 common problems (collisions and offering a help of passersby people), 17 in Metric conditions and 15 in Landmark. Next we counted 24 serious problems (grabbing, dragging, guiding by passerby people, disruption of senses, stress), 14 in Metric condition, 10 in Landmark condition.

\section{F. Recommendations for Design}

Following design recommendations for navigation instruction creation were extracted from the findings collected during the experiment: 


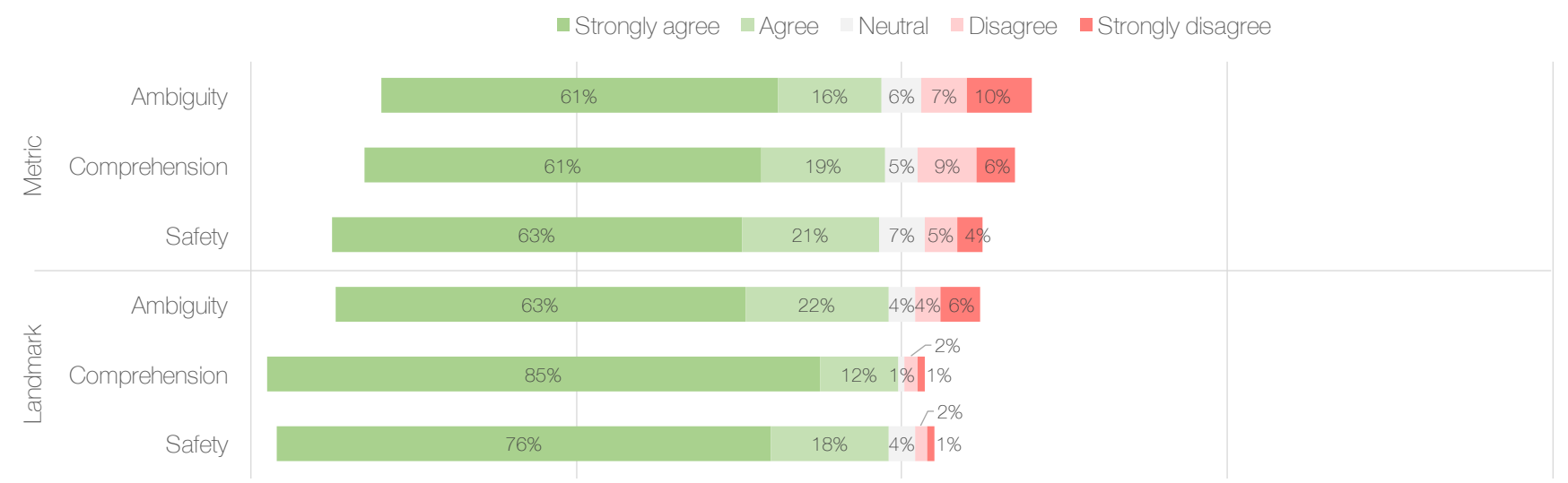

Fig. 5: Subjective judgements about level of safety, comprehension and ambiguity of navigation instructions, summarized over all segments, for both conditions $(n=16)$.

R1: The shape of a corner should be added to the itinerary if the shape is different than sharp/plain.

We observed, that participants were confused at corners, which were not sharp but bevelled or rounded.

R2: The endpoint should be added for pedestrian crossings like the pavement on the other side of the street, the opposite corner, the opposite side of the street.

The participants expected the information about the other side of the street when they used a pedestrian crossing.

R3: Change the beginning of the navigation instructions by adding a sequential number and total number of navigation instruction.

Some participants were confused when the beginning of the navigation instruction was the same one after another (i.e. crossing the street from one corner to the other corner).

R4: Changing the naming conventions for pedestrian crossings without "zebra".

One of the participants expressed concerns about usage of term "unmarked crossing" instead of "a place for crossing" for a place where there are lowered curbs but no zebra drawn on the street.

Others. Some of the participants mentioned that they would benefit from usage of GPS geofencing, which would notify them about next navigation instruction. Other participant found the Landmark version too detailed and s/he would prefer Metric version on a routes which s/he knew.

\section{Qualitative Study}

After the first experiment we implemented recommendations R1-R4 into the algorithms and geodatabase of our system. We further investigated automatically generated itineraries in a different, much more complicated urban environment (busy streets, park, passages).

\section{A. Participants}

Six visually impaired participants ( 3 female, 3 male) were recruited via e-mail leaflet sent to a group of our long term collaborators of our University. The participants in the

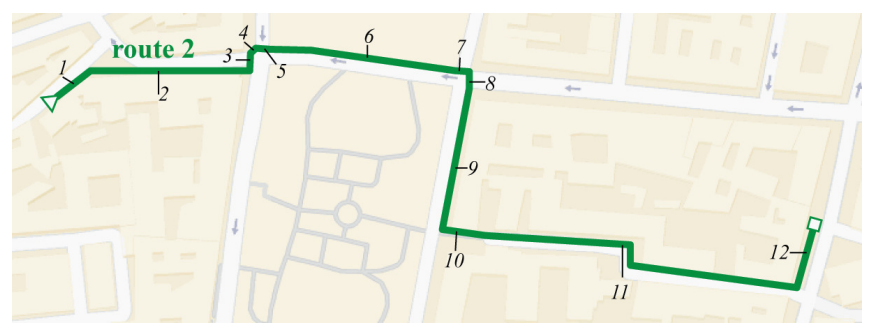

Fig. 6: Route used in qualitative study, the triangle depicts the beginning of a route, the square depicts destination of a route. Numbers represents segments numbers.

experiment were aged from 30 to 68 years $($ mean $=48.67$, $S D=15.74)$. Three participants had Category 5 visual impairment (no light perception; 3 participants had Category 4 visual impairment (light perception); 4 participants were late blind, 2 participants were congenitally blind. All of the participants were native Czech speakers. Originally we recruited 7 participants however participant P02 canceled the appointment.

\section{B. Apparatus}

Route. For our experiment we selected a route in city center outdoor environment. The route went through busy square in a city center of Prague and ended in a quiet area (see Fig. 6). The route was 670 meters long and consisted of 12 segments and 13 decision points. There were 4 pedestrian crossings on a route.

Equipment and Data collection. The equipment and the data collection were the same as in the first Comparative Study (see IV).

\section{Procedure}

The experiment consisted of one walkthrough of the route and the whole session lasted about 45 minutes. At the beginning the experimenter guided the participant to the beginning of the route, explained the purpose of 
the experiment and explained operation of the navigation application. The participants were asked to use think-aloud protocol. The task given to the participants was: "You stand in front of your house on an address Na Zborenci 276/14. To reach the destination use the navigation application. Proceed as if you were alone, but we will be watching you for your safety from a distance, we will also assist you on pedestrian crossings if needed" Then the participant started out.

When the participant reached the destination s/he was asked out his/her subjective judgment about level of comfort ("Was the navigation instruction comfortable for the participant?"), efficiency ("Did the participant think s/he proceeded efficiently?") and safety ('Did the participant feel safe?") (Likert scale 1-5 was used). The factors of subjective judgement were selected differently from the Comparative Study (see IV) as the subjective evaluation was done per route not per segment. After the experiment the participant was debriefed and received their payments.

\section{Results and Discussion}

All participants reached the destination successfully without any serious problems.

At the second decision point (bevelled corner) two participants missed the corner and described it as "unclear", P03, P04, however they found out that they missed the corner themselves after few meters.

At the second pedestrian crossing (fifth decision point) all participants turned right to face the pedestrian crossing even though they were not asked to. The next navigation instruction asked the participant to turn right, however this did not confused P07, P03, P01. On the other hand P06 and P05 crossed the pedestrian crossing immediately without selecting next navigation instruction, the did it on the other side of the street and they got confused by instruction to turn right and cross the street. P04 reported this as "weird" but was not confused by it.

At the sixth segment (pavement around greenery) all participants followed a curb on a left side along grass even though they were asked to have street on their right hand. P03 described that s/he used street as acoustic landmark on the right and followed curb on the left hand side.

Five participants found entrance to passage without any problems. Only P06 missed the passage and reported that s/he did not hear it. Participants P06 and P04 missed information about slope in a passage (it was steeply uphill).

The most problematic part of the route was segment 11 . The problematic part was "the street is bend twice". Five participants reported that they were not sure they did perceive the second bent. P03 and P07 requested directions of the bends to be present in the itinerary. P01 did not have any problems with the bends.

Surprisingly all participants found destination in the middle of the block of buildings ( 30 meters from the corner) within 5 meter precision.

Subjective judgement. After the experiment we asked the participant about their subjective judgement of efficiency,

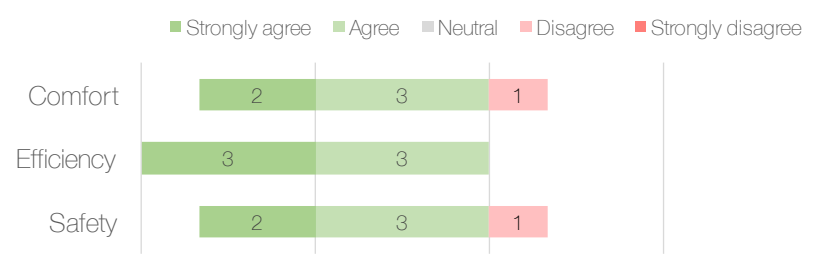

Fig. 7: Subjective judgements about level of safety, efficiency and comfort of navigation instructions $(n=6)$.

comfort and safety during the walkthrough. Fig. 7 shows that $33 \%$ of the participants strongly agreed on comfort, $50 \%$ of the participants strongly agreed on efficiency and $33 \%$ of the participants strongly agreed on safety of the navigation instructions. One participant disagreed on safety due to malfunction of acoustic signalisation on a crossing.

\section{E. Recommendations for Design}

Following design recommendations were extracted from the findings collected during the experiment:

R5: If the corner is less than 90 degrees use "slightly right/left", if the corner is more than 90 degrees use "sharply right/left".

Some participants did not recognize the corner between first and second segment of a route because the angle was not 90 degrees.

R6: If the pavement does not follow the building on one side, mention land-use on both sides (i.e. "there is greenery on your left hand side, there is street on your right hand side").

The participants followed curb along greenery and not the the street. They mentioned they would prefer knowing both sides of the pavement at this segment of the route.

R7: If there are bends on a street mention them and add directions "first right, second left".

The most problematic part of the route was where the street bends twice. Many participants did not perceive the second bend of the street.

R8: Mention pavement slope in passages.

The participants missed description of a pavement slope in a passage.

\section{CONCLUSIONS}

We developed a method for automatic generation of landmark-enhanced navigation instruction for blind pedestrians, which is based on modification of GIS data structures and development of algorithm for routing and navigation instruction generation in natural language. We conducted a comparative study of landmark-enhanced navigation instruction (Landmark) with metric-based navigation instruction (Metric) with 16 visually impaired participants. Although previous studies [8] show that landmark-based navigation is better for pedestrian navigation, the measured results were inconclusive. However subjective evaluation suggested preference of landmark-enhanced condition. Moreover we provide a 
set of design recommendations for creation of navigation instructions related mainly to corners and crossings.

Further we investigated improved landmark-enhanced method (Landmark) in qualitative study with 6 visually impaired participants resulting in additional recommendations related to leading lines or passages. Subjective evaluation suggested acceptance of the users in levels of safety, effectivity and comfort.

In the future, we will focus on integrating more landmarks such as recessed buildings or traffic sounds.

\section{ACKNOWLEDGMENT}

This research has been supported by the project Navigation of handicapped people funded by grant no. SGS16/236/OHK3/3T/13 (FIS 161 - 1611663C000) and by the Technology Agency of the Czech Republic through project Route4all (TA04031574).

\section{REFERENCES}

[1] R. G. Golledge, "Geography and the disabled: a survey with special reference to vision impaired and blind populations," Tran. of the Inst. of British Geographers, pp. 63-85, 1993. http://dx.doi.org/10.2307/623069

[2] D. Clark-Carter, A. Heyes, and C. Howarth, "The efficiency and walking speed of visually impaired people," Ergonomics, vol. 29, no. 6, pp. 779-789, 1986. http://dx.doi.org/10.1080/00140138608968314

[3] R. W. White and P. Grant, "Designing a visible city for visually impaired users," Proc. of the 2009 Int. Conf. on Inclusive Design, 2009.

[4] J. M. Loomis, R. G. Golledge, and R. L. Klatzky, "Navigation system for the blind: Auditory display modes and guidance," Presence: Teleoperators and Virtual Environments, vol. 7, no. 2, pp. 193-203, 1998. http://dx.doi.org/10.1162/105474698565677

[5] J. Armstrong, "Evaluation of man-machine systems in the mobility of the visually handicapped," Human factors in health care, pp. 331-343, 1975.

[6] A. W. Siegel and S. H. White, "The development of spatial representations of large-scale environments." $A d v$. in child development and behavior, vol. 10, p. 9, 1975. http://dx.doi.org/10.1016/S0065-2407(08)60007-5

[7] A. J. May, T. Ross, S. H. Bayer, and M. J. Tarkiainen, "Pedestrian navigation aids: information requirements and design implications,' Personal and Ubiquitous Computing, vol. 7, no. 6, pp. 331-338, 2003. http://dx.doi.org/10.1007/s00779-003-0248-5

[8] T. Ross, A. May, and S. Thompson, "The use of landmarks in pedestrian navigation instructions and the effects of context," in MobileHCI 2004. Springer, 2004, pp. 300-304. http://dx.doi.org/10. 1007/978-3-540-28637-0_26
[9] K. Rehrl, E. Häusler, and S. Leitinger, "Comparing the effectiveness of GPS-enhanced voice guidance for pedestrians with metric-and landmark-based instruction sets," in Geographic information science. Springer, 2010, pp. 189-203. http: //dx.doi.org/10.1007/978-3-642-15300-6_14

[10] A. Millonig and K. Schechtner, "Developing landmark-based pedestrian-navigation systems," ITSC 2007, vol. 8, no. 1, pp. 43-49, 2007. http://dx.doi.org/10.1109/TITS.2006.889439

[11] S. Millar, Understanding and representing space: Theory and evidence from studies with blind and sighted children. Oxford University/Clarendon Press, 1994.

[12] S. Millar, Space and sense. Psychology Press, 2008

[13] C. Thinus-Blanc and F. Gaunet, "Representation of space in blind persons: vision as a spatial sense?" Psychological bulletin, vol. 121, no. 1, p. 20, 1997. http://dx.doi.org/10.1037/0033-2909.121.1.20

[14] N. Raz, E. Striem, G. Pundak, T. Orlov, and E. Zohary, "Superior serial memory in the blind: a case of cognitive compensatory adjustment," Current Biology, vol. 17, no. 13, pp. 1129-1133, 2007. http://dx.doi.org/10.1016/j.cub.2007.05.060

[15] N. A. Bradley and M. D. Dunlop, "An experimental investigation into wayfinding directions for visually impaired people," Personal and Ubiquitous Computing, vol. 9, no. 6, pp. 395-403, 2005. http://dx.doi.org/10.1007/s00779-005-0350-y

[16] M. Bujacz, P. Baranski, M. Moranski, P. Strumillo, and A. Materka, "Remote guidance for the blind - a proposed teleassistance system and navigation trials," in HSI 2008. IEEE, 2008, pp. 888-892. http://dx.doi.org/10.1109/HSI.2008.4581561

[17] J. Faria, S. Lopes, H. Fernandes, P. Martins, and J. Barroso, "Electronic white cane for blind people navigation assistance," in WAC 2010. IEEE 2010, pp. 1-7.

[18] H. Petrie, V. Johnson, T. Strothotte, R. Michel, A. Raab, and L. Reichert, "User-centred design in the development of a navigational aid for blind travellers," in INTERACT 1997. Springer, 1997, pp 220-227. http://dx.doi.org/10.1007/978-0-387-35175-9_39

[19] M. Pielot, B. Poppinga, W. Heuten, and S. Boll, "A tactile compass for eyes-free pedestrian navigation," in INTERACT 2011. Springer, 2011, pp. 640-656. http://dx.doi.org/10.1007/978-3-642-23771-3_47

[20] J. Vystrčil, Z. Míkovec, and P. Slavík, "Naviterier-indoor navigation system for visually impaired," 2012

[21] WHO, "ICD update and revision platform: change the definition of blindness," 2009. http://www.who.int/blindness/Changel\%20thel $\% 20$ Definition $\backslash \% 20$ of $\$$ 20Blindness.pdf

[22] V. R. Schinazi, "Spatial representation and low vision: Two studies on the content, accuracy and utility of mental representations," in Int. Congress Series, vol. 1282. Elsevier, 2005, pp. 1063-1067. http://dx.doi.org/10.1016/j.ics.2005.05.163

[23] T. Ishikawa, H. Fujiwara, O. Imai, and A Okabe, "Wayfinding with a gps-based mobile navigation system: A comparison with maps and direct experience," Journal of Environmental Psychology, vol. 28, no. 1, pp. 74-82, 2008. http://dx.doi.org/10.1016/j.jenvp.2007.09.002

[24] P. Dragicevic, "HCI statistics without p-values," 2015. 